Phase- shift al gor i thm for whi te- I ight i nt erferometry i nsensi tive to I i near errors in phase shi $\mathrm{ft}$

\begin{tabular}{|l|l|}
\hline 著者 & Adachi Nasaaki \\
\hline $\begin{array}{l}\text { j our nal or } \\
\text { publ i cat i on t i t l e }\end{array}$ & Opt i cal Revi ew \\
\hline vol une & 15 \\
\hline nunber & 3 \\
\hline page $r$ ange & 148 - 155 \\
\hline year & 2008 05- 01 \\
\hline URL & ht t p: //hdl . handl e. net /2297/9976 \\
\hline
\end{tabular}




\title{
Phase-Shift Algorithm for White-Light Interferometry Insensitive to Linear Errors in Phase Shift
}

\author{
Masaaki Adachi* \\ Graduate School of Natural Science and Technology, Kanazawa University, \\ Kakuma-machi, Kanazawa, 920-1192, Japan
}

White-light interference has changes in fringe contrast. When phase-shift techniques are applied to white-light interference, the phase-shift algorithm which can extract the phase accurately under the contrast changes is required. There is often another requirement that the phase shift between frames should not be restricted to $\pi / 2$. Computer simulations show that the well-known algorithms have non-negligible errors under both requirements. To find an algorithm which will satisfy the requirements, I extract individual terms $\left(I_{j} \mp\right.$ $I_{k}$ ) in an algorithmic equation by considering symmetry of light intensity against phase, where $I_{j}$ is light intensity just after the $j$-th phase shift. Using computer simulations, I 
search for appropriate coefficients by which the terms are multiplied in the equation, finally finding an algorithm which satisfies both the requirements with the phase shift used.

KEYWORDS: phase shift, algorithm, white-light interferometer, fringe contrast, error estimation, computer simulation

* E-mail address: adachi@t.kanazawa-u.ac.jp 


\section{Introduction}

Application of white-light interferometry is expanding in the field of optical testing of precision products. This is thought to be primarily due to the fact that the interferometry is capable of measuring three-dimensional (3-D) shapes of step-like objects, and secondly due to its high vertical resolution ${ }^{1)}$. Many kinds of micro step-like objects are newly designed and now produced as key elements of Micro Electro-Mechanical Systems (MEMS). Newly designed and test-produced parts require speedy and precise estimation of their 3-D shapes using high resolution technology. An interference microscope using white light is suitable to measure 3-D shapes of small objects like key elements related to MEMS.

In 3-D shape measurement with nanometric vertical resolution, the white-light interferometer is combined with a phase-shift technology. The combined system makes incremental vertical step movements related to phase shifting and captures interferograms. After repeating these processes one way over a measured range it searches the maximum contrast interferogram whose capturing point depends on the height at the measurement position. The searched interferograms are analyzed with a phase-shift algorithm and the 3-D shape is finally measured with the nanometric 
resolution of the phase-shift algorithm.

Used algorithms, however, have not been developed for white-light interference, but have been developed only for monochromatic light interference as far as I am aware. It was assumed that interference light intensity changes have a constant contrast. Reasons why such algorithms are used even for the white-light interference are thought to emanate from that the algorithms used do not seem to make conspicuous errors even in white-light interference; many kinds of algorithms have been developed, and numerous analyses of them have been done. In white-light interference, however, the contrast is at its maximum at the optical path difference (OPD) of zero and sharply decreases when the absolute value of OPD increases. Therefore, the phase-shift algorithm required in a white-light interferometer must have the capability of extracting phase accurately from the interference which has contrast changes.

There is often another requirement: that the algorithm should work with a phase shift other than $\pi / 2$. Because of inaccurate calibration of phase shifters, numerical aperture (NA) effect ${ }^{2}$ of an used objective lens, restricted capture conditions or others, the phase shift might differ from $\pi / 2$. Thus, there has been a great deal of research to develop algorithms using such a phase shift in monochromatic light interference. When 
the phase shift is not $\pi / 2$ but is constant, the difference from $\pi / 2$ is called a linear error in phase shift. In the phase-shift technologies combined with white-light interferometry the linear shift error seems to make a non-negligible error in phase extraction. An ideal algorithm would be insensitive to an unknown linear shift error. When it is difficult to develop the ideal algorithm, an acceptable algorithm must be insensitive to the linear shift error known, because such linear shift errors can be determined from captured interferograms ${ }^{3)}$.

In the first half of this paper, computer simulation is carried out to estimate errors which are retained by applying well-known algorithms to the white-light interference. The estimated algorithms are of 4-frame Carre ${ }^{4)}$, simple 4-frame ${ }^{5)}$, 5-frame Hariharan ${ }^{6}$, 7-frame Groot ${ }^{7)}$, 5-frame in Schmit ${ }^{8)}$, and 6-frame in Hibino ${ }^{9)}$. Among the algorithms the one satisfying the former requirement is found to be the 7-frame Groot algorithm. In fact, it has a very low error when interferograms are captured with $\pi / 2$ shift. However, under both the requirements all the estimated algorithms have non-negligible errors.

In the second half of the paper, I develop a new algorithm that satisfies both the requirements. The linear shift error described here means that the phase shift is known but is not $\pi / 2$. In the development, I use symmetry of light intensity against phase 
in white-light interference. Combining symmetry and computer simulation, I find an algorithm satisfying both the requirements.

The algorithm I find is different from the well-known basic form in which the equational expression has sine components in the numerator and cosine components in the denominator ${ }^{10)}$. In addition, it works for a high-speed precision profilometer using a phase shift greater than $2 \pi^{11)}$. This profilometer is one of white-light interferometers, but uses two extremely short-coherent light sources that have different center wavelengths, $503 \mathrm{~nm}$ and $591 \mathrm{~nm}$. In this profilometer the phase shift is calculated as $6.36 \pi$ for 503 $\mathrm{nm}$ and $5.41 \pi$ for $591 \mathrm{~nm}$. Both shifts differ from (integer $+1 / 2$ ) $\pi$. Computer simulation shows that such a large phase shift can be used with the algorithm found.

\section{Error Estimations of Representative Algorithms for White}

\section{-Light Interference}

One can find many phase-shift algorithms in published papers. They were developed for real fringes including different kinds of errors. Some are insensitive to a linear error in phase shift, some are insensitive to non-linear errors in phase shift. All are 
developed under the assumption that interference fringes have a constant contrast. However, there is a possibility that some of them can accurately extract phase even of white-light interference. Therefore, error estimations should be made if they are applied to white-light interference.

Estimations are made on 6 representative phase-shift algorithms. They are 4-frame Carre ${ }^{4)}$, simple 4-frame ${ }^{5)}$, 5-frame Hariharan ${ }^{6)}$, 7-frame Groot ${ }^{7)}$, 5-frame in Schmit $^{8)}$, and 6-frame in Hibino ${ }^{9)}$ algorithms. There are many other algorithms and still others may be in development ${ }^{12)}$, but they are not identified here, because of restricted space in the paper.

In error estimations, I use a computer to make simple light-intensity changes having contrast changes and noise. Light intensities $I_{j}$ expressed by the following are used,

$$
I_{j}=A+B \exp \left(-E \cdot \varphi_{j}{ }^{2}\right) \cos \varphi_{j}+N \cdot R_{j},
$$

where $j$ is sampling number, $A$ and $B$ are constants related to the reference and object light intensities, and $A$ works as an offset. $E$ characterizes the contrast changes, $\varphi_{j}$ is 
phase, $N$ characterizes noise intensity, and $R_{j}$ is a random number whose statistical distribution is uniform from -0.5 to 0.5 . Phase $\varphi_{j}$ is related to OPD at the $j$-th sampling moment, $O P D_{j}$, by the next equation,

$$
\varphi_{j}=2 \pi \frac{O P D_{j}}{\lambda}
$$

where $\lambda$ is the center wavelength of an extremely short-coherent light.

\subsection{4-frame Carre algorithm ${ }^{4)}$}

The Carre algorithm is the oldest one that is insensitive to a linear error in phase shift $\alpha$. This algorithm is described by eq. (3),

$$
\begin{aligned}
& \varphi_{C}=\arg \left(i \left\{\left[3\left(I_{2}-I_{3}\right)-\left(I_{1}-I_{4}\right)\right]\right.\right. \\
& \left.\left.\square\left(I_{1}-I_{4}+I_{2}-I_{3}\right)\right\}^{1 / 2}+\left(I_{2}+I_{3}-I_{1}-I_{4}\right)\right)
\end{aligned},
$$

where $i$ means the unit imaginary and $I_{j}$ are given from eq. (1) by changing $\varphi_{j}$ with the next equation, 


$$
\varphi_{j}=\varphi_{0}+\frac{\alpha}{2}(2 j-5) ; j=1 \square 4
$$

When light intensity change is purely sinusoidal $(E=N=0)$, phase error $\left(\varphi_{C}-\varphi_{0}\right)$ of the Carre algorithm becomes zero. To check the performance of computer simulation the phase errors of the Carre algorithm are estimated. They are shown in Fig. 1. $X$ axis is phase shift $\alpha, y$ axis is phase $\varphi_{0}$ in eq. (4), and $z$ axis is phase error given as $\left(\varphi_{C}-\varphi_{0}\right) / 2 \pi$ in \%. The right white line shows the errors at $\alpha$ which is near $0.7 \pi$, the middle white line shows those of $\alpha=0.5 \pi$, and the left white line shows those near $0.3 \pi$. The simulated errors calculated at all the points are zero as expected.

Next, I introduce light intensity changes due to extremely short-coherent light. This intensity is easily given by setting $E$ as non-zero in eq. (1). The light intensity changes calculated using $E=0.1$ and $N=0$ are shown in Fig. 2 . The $x$ axis is $\varphi_{0}$ and the intensity changes are calculated at $\varphi_{0}$ from $-6 \pi$ to $6 \pi$. The Carre algorithm is tentatively applied to the intensities sampled with phase shift $\alpha=0.5 \pi$. At $\varphi_{0}$ from $-6 \pi$ to $6 \pi$, the estimated phase errors $\left(\varphi_{C}-\varphi_{0}\right) / 2 \pi$ in $\%$ are shown along the $y$ axis in Fig. 3. When light having the center wavelength of $\lambda=600 \mathrm{~nm}$ is used, $5 \%$ of the phase error equates with a height error of $15 \mathrm{~nm}(=300 \mathrm{~nm} \times 0.05)$. These are only the case for $\alpha=0.5 \pi$. Error 
estimations are then done at other $\alpha$ between $0.3 \pi$ to $0.7 \pi$. The estimated errors are shown in Fig. 4. We cannot obtain experimental signals without noise. In addition to the above contrast changes, noise is then introduced by setting $N$ as 0.03 in eq. (1). The errors due to $E=0.1$ and $N=0.03$ are shown in Fig. 5 .

\subsection{Simple 4-frame algorithm ${ }^{5}$}

This is also called the conventional 4-frame algorithm. The phase $\varphi_{S-4}$ extracted by the algorithm is given by eq. (5),

$$
\varphi_{S-4}=\arg \left[i\left(I_{4}-I_{2}\right)+\left(I_{1}-I_{3}\right)\right] \text {, }
$$

where $I_{j}$ is given from eq. (1) by changing $\varphi_{j}$ with the next equation,

$$
\varphi_{j}=\varphi_{0}+\alpha(j-1) \quad ; j=1 \square 4
$$

When the contrast changes and noise are introduced in eq. (1) by setting $E=0.1$ and $N=0.03$, the estimated phase errors $\left(\varphi_{S-4}-\varphi_{0}\right)$ are shown in Fig. 6. Compared with Fig. 5, 
the errors due only to noise are smaller, but the error dependence on phase $\varphi_{0}$ is bigger, and the error dependence on the linear shift error given by $\alpha-0.5 \pi$ is also bigger.

\subsection{5-frame Hariharan algorithm ${ }^{6}$}

The 5-frame Hariharan algorithm is known as an error-immune algorithm.

The algorithm is classified as one of the averaging algorithms ${ }^{8)}$. The phase $\varphi_{5-H}$ is calculated by eq. (7a),

$$
\begin{aligned}
& \varphi_{5-f}=\arg \left\{2 i\left(I_{2}-I_{4}\right)\right. \\
& \left.+\left[2 I_{3}-\left(I_{1}+I_{5}\right)\right]\right\}
\end{aligned}
$$

Introducing a simple correction for a linear shift error, eq. (7a) can be rewritten as the next equation,

$$
\begin{aligned}
& \varphi_{5-H}^{\prime}=\arg \left\{2 i \sin \alpha\left(I_{2}-I_{4}\right)\right. \\
& \left.+\left[2 I_{3}-\left(I_{1}+I_{5}\right)\right]\right\}
\end{aligned}
$$

where $I_{j}$ is given from eq. (1) by changing $\varphi_{j}$ with the next equation, 


$$
\varphi_{j}=\varphi_{0}+\alpha(j-3) \quad ; j=1 \square 5
$$

When the same intensity of contrast changes and the same intensity of noise ( $E=0.1$ and $N=0.03)$ are introduced, the phase errors $\left(\varphi_{5-H}^{\prime}-\varphi_{0}\right)$ are estimated and are shown in Fig. 7. Compared with Fig. 6, the estimated errors are less, and the error dependence on the linear shift error is also less.

\subsection{7-frame Groot algorithm ${ }^{7)}$}

The 7-frame Groot algorithm is more famous as an error-immune algorithm.

The phase $\varphi_{7-G}$, calculated by the 7-frame Groot algorithm, is given as the following,

$$
\begin{aligned}
& \varphi_{7-G}=\arg \left\{i\left[7\left(I_{2}-I_{4}\right)-\left(I_{0}-I_{6}\right)\right]\right. \\
& \left.+\left[8 I_{3}-4\left(I_{1}+I_{5}\right)\right]\right\}
\end{aligned}
$$

Introducing a simple correction for a linear shift error, eq. (9a) can be rewritten as the next equation, 


$$
\begin{aligned}
& \varphi_{7-G}^{\prime}=\arg \left\{2 i \sin \alpha\left[7\left(I_{2}-I_{4}\right)-\left(I_{0}-I_{6}\right)\right]\right. \\
& \left.+\left(1+\sin ^{2} \alpha\right)\left[8 I_{3}-4\left(I_{1}+I_{5}\right)\right]\right\}
\end{aligned},
$$

where $I_{j}$ is given from eq. (1) by changing $\varphi_{j}$ with the next equation,

$$
\varphi_{j}=\varphi_{0}+\alpha(j-3) ; j=0 \square 6 \text {. }
$$

When the same intensity of contrast changes and the same intensity of noise ( $E=0.1$ and $N=0.03)$ are introduced, the phase errors $\left(\varphi_{7-G}^{\prime}-\varphi_{0}\right)$ are shown in Fig. 8. Compared with Fig. 7, the estimated errors are in the same order, but the error dependence on $\varphi_{0}$ is smoother, and the error fluctuation within a local area is also smaller.

\subsection{5-frame in Schmit algorithms ${ }^{8)}$ and 6-frame in Hibino algorithms $^{9)}$}

Schmit and Creath, ${ }^{8)}$ and Hibino et al ${ }^{9)}$ proposed many algorithms which are distinguished by the number of used frames and derivative forms. Among them I arbitrarily select eq. (11) as the 5-frame of Schmit algorithms, 


$$
\begin{aligned}
& \varphi_{5-S}=\arg \left\{i\left[\left(I_{1}-I_{5}\right)+4\left(I_{4}-I_{2}\right)\right],\right. \\
& \left.+\left[6 I_{3}-2\left(I_{4}+I_{2}\right)-\left(I_{1}+I_{5}\right)\right]\right\}
\end{aligned},
$$

where $I_{j}$ is given from eq. (1) by changing $\varphi_{j}$ with the next equation,

$$
\varphi_{j}=\varphi_{0}+\alpha(j-3) ; j=1 \square 5
$$

Among Hibino algorithms, I arbitrarily select the 6-frame algorithm given by the next equation,

$$
\begin{aligned}
& \varphi_{6-H}=\arg \left\{\sqrt{3} i\left[5\left(I_{1}-I_{6}\right)-6\left(I_{2}-I_{5}\right)-17\left(I_{3}-I_{4}\right)\right]\right. \\
& \left.+\left[\left(I_{1}+I_{6}\right)-26\left(I_{2}+I_{5}\right)+25\left(I_{3}+I_{4}\right)\right]\right\}
\end{aligned}
$$

where $I_{j}$ is given from eq. (1) by changing $\varphi_{j}$ with the next equation ( $\alpha$ is around $\pi / 3$ ),

$$
\varphi_{j}=\varphi_{0}+\alpha\left(j-\frac{7}{2}\right) ; j=1 \square 6
$$

When only the same intensity of noise $(N=0.03)$ is introduced, the phase errors $\left(\varphi_{5-S}-\varphi_{0}\right)$ 
and the phase errors $\left(\varphi_{6-H}-\varphi_{0}\right)$ are calculated, respectively. They are very small as expected. But, when the same intensity of contrast changes $(E=0.1)$ is introduced, the phase errors $\left(\varphi_{5-S}-\varphi_{0}\right)$ and the phase errors $\left(\varphi_{6-H}-\varphi_{0}\right)$ become pretty large, especially at lower values of $\alpha$. They are not shown here, but the maximum estimated errors are on the same order as those of Fig. 8.

In this chapter the 6 representative algorithms were estimated. A zero phase error shown in Fig. 1 is truly desired. However, all of the 6 representative algorithms involve non-negligible errors due to contrast changes. In these estimations the 7-frame Groot algorithm has very little errors, only at phase shift $\alpha=0.5 \pi$. This error dependence on phase $\varphi_{0}$ is illustrated by the middle white line in Fig. 8 . When $\alpha$ moves farther away from $\pi / 2$ the phase errors become larger. The error dependence on phase $\varphi_{0}$ at $\alpha$ near $0.3 \pi$ and of $0.7 \pi$ are also illustrated by white lines in Fig. 8 .

\section{New Algorithm Insensitive to Contrast Changes and also to}

\section{Linear Error in Phase Shift}

The 6 representative algorithms which use fewer or equal number of 7 frames 
have been estimated above. Algorithms using more frames would become more complex and seem more time-consuming regarding calculation. Within 7 frames it is conceivable that there are many other algorithms which have not been investigated by anyone. Some of them might be insensitive to contrast changes and also to the linear error in phase shift. Here, I begin to develop a new algorithm with the extraction of individual terms $\left(I_{j} \mp I_{k}\right)$ in an algorithmic equation by considering symmetry of light intensity against phase $\varphi_{0}$.

When light intensities are sampled around a contrast peak, one group of sampled $I_{j}(j$ is from 0 to 6 ) are given as white circles in Fig. 9. The phase calculated from these intensities is herein assumed to be $\varphi_{3}$ of $I_{3}$, the middle point in the group. Apart from this group let us consider another group of sample points given by $I_{j}^{\prime}$. The latter group is positioned as having $y$-axis symmetry to the former group and is represented as black circles in Fig. 9. The suffixes of $I_{j}$ and of $I_{j}^{\prime}$ are not symmetric, but become bigger on the right. The next equations are concluded from the symmetry.

$$
\begin{array}{ll}
I_{0}=I_{6}^{\prime} & \\
I_{1}=I_{5}^{\prime} & I_{4}=I_{2}^{\prime} \\
I_{2}=I_{4}^{\prime}, & I_{5}=I_{1}^{\prime} . \\
I_{3}=I_{3}^{\prime} & I_{6}=I_{0}^{\prime}
\end{array}
$$


In addition, the phase of $I_{3}$ calculated from the group of $I_{j}$ and the phase of $I_{3}^{\prime}$ calculated from the group of $I_{j}^{\prime}$ should be equal in absolute value, but different in sign. Then, let us consider the next equation,

$$
\begin{aligned}
& \varphi_{N}=\arg \left\{i \left[a\left(I_{2}-I_{4}\right)+b\left(I_{1}-I_{5}\right)\right.\right. \\
& \left.+c\left(I_{0}-I_{6}\right)\right]+\left[8 I_{3}+d\left(I_{2}+I_{4}\right)\right. \\
& \left.\left.+e\left(I_{1}+I_{5}\right)+f\left(I_{0}+I_{6}\right)\right]\right\}
\end{aligned}
$$

where the imaginary part is a polynomial of differential terms and the real part is a polynomial of addition terms. As the differential terms change their sign by symmetric transformation and the additional terms do not change their sign, the phase symmetry denotes that the phase should be expressed by eq. (16) as a simple case. Even in this simple equation, the number of independent variables $(a, b, c, d, e, f)$ is 6 . There are many variables to narrow this form down to the required equation. So, let us also suppose that eq. (16) should extract the phase correctly under well-used experimental conditions. As the first condition, an offset of light intensity should not affect the phase. The polynomial of differential terms cannot have offsetting effects, nor should the polynomial of the addition terms involve the offset. This necessitates the next equation, 


$$
8+2 d+2 e+2 f=0
$$

As the second condition, substituting the purely sinusoidal intensity changes sampled with $\pi / 2$ shift to eq. (16) must extract the phase correctly. This requirement gives the next relation:

$$
2(a-c)=8-2 e .
$$

From eqs. (17) and (18) the number of independent variables becomes 4 , I set them as $b$, $c, d$, and $e$. These 4 variables determine $a$ and $f$. There are still many variables, so that I cannot approach proper values for variables or coefficients by numerical simulation. Here, let us consider again eqs. (7a) and (9a). Their real parts are the same and only their imaginary parts are different. By focusing on the imaginary parts, $c$ in eq. (16) seems to control the resemblance ratio between the 5-frame Hariharan and the 7-frame Groot. For example, in the case where a purely sinusoidal intensity change is sampled with $\pi / 2$ shift, the $b\left(I_{1}-I_{5}\right)$ term has no effect. Therefore, $c=0$ means that the imaginary part in eq. (16) 
becomes the same as the 5 -frame, and $c=-1$ means the imaginary part becomes similar to the 7-frame. If $e$ is set as -4 in eq. (16), the real and imaginary parts are exactly the same as that of the 5 -frame or the 7-frame, because $c=0$ and $e=-4$ lead $a=8$ by eq. (18) resulting eq. (16) becomes eq. (7a), and $c=-1$ and $e=-4$ lead $a=7$ resulting eq. (16) becomes eq. (9a). As the 5-frame Hariharan and the 7-frame Groot are famous algorithms having error immunity, it is natural to investigate an algorithm similar to them. Therefore, let me set $e$ as -4 .

From eqs. (17) and (18), setting $e=-4$ means $f=-d$, and $a=8+c$. The independent variables have been narrowed to $b, c$, and $d$. Then, with $e=-4$ and $b=d=0$, phase errors at phase shift $\alpha=0.7 \pi$ are estimated by changing $c$ to investigate its effect. The examples of the estimated phase errors are shown in Fig. 10. The solid curve is given by $c=-1$ and is just the 7-frame. The broken curve is given by $c=0$ and is just the 5 -frame. The dotted curve is given by $c=-2$. This solid curve approximately equals the right white line in Fig.8 viewed from the $-x$ point. From Figs. 7, 8, 10 and some simulations, changing $c$ does not seem to reduce the phase errors greatly. From Fig. 10 a good value for $c$ seems to be around -1 .

I have not yet investigated the independent variables $b$ and $d$. These terms have 
not been included in basic formulas in the phase-shift algorithms ${ }^{10}$, the 5 -frame Hariharan $^{6)}$ or the 7 -frame Groot ${ }^{7)}$. Here, I estimate the phase errors by changing only $b$, and also by changing only $d$. In Fig. 11 the estimated phase errors are shown. The solid curve is just the 7-frame ( $c=-1, b=d=0$ ). The broken curve is $c=-1, b=-5$, and $d=0$, and the dotted curve is of $c=-1, b=0$, and $d=-4.5$. The broken curve and the dotted curve have opposite-sign differences in a local area from the solid curve, but the base lines of both curves become less sloped in the entire area compared with the solid curve. Therefore, I searched $b$ and $d$ to minimize phase errors and found $b=-22$, and $d=-14.5$. These values give the errors as shown in Fig. 12. This is obtained for the phase shift $\alpha=0.7 \pi$.

Next, I searched $b$ and $d$ to minimize phase errors for $\alpha=0.3 \pi$ and found $b=23$ and $d=14$. The minimized phase errors are shown in Fig. 13. In addition, I found appropriate values $b$ and $d$ which minimize the phase errors for any values of phase shift $\alpha$ between $0.3 \pi$ and $0.7 \pi$. The values found are shown in Fig. 14 . This shows that a linear relation might exist between $b$ and $d$, and the ratio of $b$ to $d$ is found as $b / d=1.6$. Using this ratio I estimate the phase errors for the phase shift $\alpha$ between $0.3 \pi$ and $0.7 \pi$ by changing $d$ as given in Fig. 14. Figure 15 shows the minimized phase errors. An inset figure shows the used intensity changes having contrast changes of $E=0.1$. Comparing 
this figure with Fig. 7 or Fig. 8 the obtained phase errors are reduced substantially for any $\alpha$ between $0.3 \pi$ and $0.7 \pi$.

The above ratio $b / d=1.6$ is found only for the contrast changes of $E=0.1$. I next estimate the ratio for other contrast changes of $E \neq 0.1$. Finally, it is discovered that the ratio $b / d=1.6$ and $d$ given by Fig. 14 are valid for other values of $E \leq 0.15$. Figure 16 shows the reduced phase errors given for $E=0.15$. The inset figure shows the intensity changes used and is quite different from that in Fig. 15. This validity means that $b$ and $d$ in Fig. 14 are important. In this figure, $b$ and $d$ seem to take simple and symmetrical changes around $\alpha=0.5 \pi$. Then I try to approximate $d$ as an equation of $\alpha$. After some trials $d$ is found to be approximately expressed by the next equation,

$$
d \cong-4.1\left(\alpha-\frac{\pi}{2}\right)-71\left(\alpha-\frac{\pi}{2}\right)^{5}
$$

For $\alpha(0.4 \pi<\alpha<0.6 \pi), d$ could be expressed only by the first term. With eq. (19), the required algorithm is finally expressed by the next equation. 


$$
\begin{aligned}
& \varphi_{N}=\arg \left\{i \left[7\left(I_{2}-I_{4}\right)+1.6 d\left(I_{1}-I_{5}\right)\right.\right. \\
& \left.-\left(I_{0}-I_{6}\right)\right]+\left[8 I_{3}+d\left(I_{2}+I_{4}-I_{0}-I_{6}\right) .\right. \\
& \left.\left.-4\left(I_{1}+I_{5}\right)\right]\right\}
\end{aligned}
$$

As mentioned in $\S 1$, a plofilometer that uses a phase shift greater than $2 \pi$ was

developed to do high-speed vertical scanning ${ }^{11)}$. Its interferometer uses two extremely short-coherent light sources that have different center wavelengths, $503 \mathrm{~nm}$ and $591 \mathrm{~nm}$. A phase step is calculated as $6.36 \pi$ for $503 \mathrm{~nm}$ and $5.41 \pi$ for $591 \mathrm{~nm}$. Both phase shifts differ from (integer $+1 / 2) \pi$. Then, I estimate the residual phase errors that the found algorithm is applied to for intensities sampled with a phase shift greater than $2 \pi$. Figure 17 shows the residual phase errors with a phase shift $\alpha$ between $6.3 \pi$ and $6.7 \pi$. In this estimation $E=0.008$ is used to obtain nearly the same half width of contrast changes along sampled suffixes $j$ as that of the intensity changes calculated with $E=0.1$, and a fraction of $\alpha^{\prime}\left(\alpha^{\prime}=\alpha-6 \pi\right)$ is used instead of $\alpha$ to obtain $d$.

\section{Discussion and Considerations}

This paper first estimates the combined effects of $b\left(I_{1}-I_{5}\right)$ and $d\left(I_{2}+I_{4}\right)$ terms in a phase-shift algorithm. When sinusoidal intensity changes of a constant contrast are 
sampled with $\pi / 2$ phase shift, these terms have no effect. Thus, it is natural to consider formulas which do not include $b\left(I_{1}-I_{5}\right)$ or $d\left(I_{2}+I_{4}\right)$. I can find only a rare equation which includes $\left(I_{1}-I_{5}\right)$ and $\left(I_{2}+I_{4}\right)$ terms. This equation is the 5 -frame from Schmit ${ }^{8)}$ which has the ratio between $b$ and $d$ of $-1 / 2$. This ratio is different from the proposed ratio of 1.6. When white light interference is sampled with phase shift $\alpha \neq \pi / 2, b\left(I_{1}-I_{5}\right)$, and $d\left(I_{2}+I_{4}\right)$ terms have effects on phase extraction. Although these effects depend on $\alpha$, respectively, I found that the relation $b / d=1.6$ gives the minimized phase errors in the range of $\alpha$ from $0.3 \pi$ to $0.7 \pi$. It is also important that this ratio does not change over some other values of $E \leq 0.15$, characterizing parameter of contrast changes.

There is a possibility that the $b / d$ ratio might depend on the shapes of envelope curves. I therefore estimate the effect of shapes of envelope curves. An envelope curve is given by the Fourier transformation of the specific spectral curve of light used. When a spectral curve of light is Gaussian, the envelope curve is also given as Gaussian. Many kinds of light sources are commonly approximated to have a Gaussian spectrum. The Gaussian curve expressed as $\exp \left(-E \varphi^{2}\right)$ is used herein. I estimate phase errors due to envelope curves expressed as $\exp \left(-E|\varphi|^{1.5}\right)$ and $\exp \left(-E|\varphi|^{2.5}\right)$, respectively, with computer simulations. The phase errors extracted for both of the envelope curves using the ratio 
$b / d=1.6$ and $d$ given in Fig. 14, are nearly the same as that for $\exp \left(-E|\varphi|^{2}\right)$, showing the relation $b / d=1.6$ and $d$ in Fig. 14 work even in non-Gaussian envelope curves.

In real applications, a certain intensity of noise is always involved in signals. Thus, noise immunity should be estimated in this algorithm. For this estimation $N$ in eq. (1) is set at 0.03 , and phase errors are calculated using these noise signals. Figures 15-17 show the estimated phase errors of $N=0.03$. To compare these results with Fig. 8 of the 7-frame algorithm which is reported as a vibration and noise-immune algorithm ${ }^{7)}$, the vertical distribution widths of the phase errors due to the noisy signals are all nearly the same. Therefore, this algorithm is thought to have noise-immunity.

In the new algorithm development described above, $e=4$ is used to simplify the computer simulation. The condition of $e=4$ comes from analogy with the 5 -frame Hariharan or the 7-frame Groot algorithms. There is a possibility that other values of $e$ have good performance in phase extraction. I am researching this now.

\section{Conclusions}

White-light interference involves changes in fringe contrast. To obtain a 
precise phase of white-light interference a phase-shift algorithm in which the extracted phase is insensitive to changes in contrast is required. There is often another requirement that phase shift between captured interferograms must not be restricted to $\pi / 2$. Therefore, a phase-shift algorithm that is insensitive both to contrast changes and to a linear error in the phase shift is required. Computer simulation showed that the well-known representative algorithms developed thus far cannot satisfy both these requirements. Therefore, I started to develop a new algorithm by extracting individual terms $\left(I_{j} \mp I_{k}\right)$ in the algorithmic equation by considering symmetry of light intensity change against phase. Using computer simulation again, I searched for appropriate coefficients by which the terms are multiplied in the equation. I finally found an algorithm satisfying both the necessary requirements. It was found to have the same noise-immunity as representative noise-immune algorithms and to be independent of changing intensity for the fringe contrasts. In addition, this algorithm can be applied to the light intensities sampled with phase shift $\alpha$ greater than $2 \pi$. 


\section{References}

1) K. G. Larkin: J. Opt. Soc. Am. A 13 (1996) 832.

2) K. Creath: Appl. Opt. 28 (1989) 3333.

3) J. Schmit and A. Olszak: Appl. Opt. 41 (2002) 5943.

4) J. E. Greivenkamp and J. H. Bruning: in Optical Shop Testing, ed. D. Malacara (Wiley, New York, 1992) 2nd ed., p. 524.

5) J. E. Greivenkamp and J. H. Bruning: in Optical Shop Testing, ed. D.Malacara (Wiley, New York, 1992) 2nd ed. , p. 510.

6) P. Hariharan, B. F. Oreb, and T. Eiju: Appl. Opt. 26 (1987) 2504.

7) P. de Groot: Appl. Opt. 34 (1995) 4723.

8) J. Schmit and K. Creath: Appl. Opt. 34 (1995) 3610.

9) K. Hibino, B. F. Oreb, D. I. Farrant, and K. G. Larkin: J. Opt. Soc. Am. A 14 (1997) 918.

10) Y. Surrel: Appl. Opt. 32 (1993) 3598.

11) M. Adachi: Proc. SPIE 5602 (2004) 73.

12) A. Patil, B. Raphael, and P. Rastogi: Opt. Let. 29 (2004) 1381. 


\section{Figure captions}

Fig. 1: Phase errors estimated for the 4-frame Carre algorithm ${ }^{4)}$. Interference light intensity changes are assumed to have a constant contrast and no noise. The errors are zero as expected.

Fig. 2: Typical interference light intensity changes of white-light interference. These changes are calculated with eq. (1) using $E=0.1$ and $N=0$.

Fig. 3: Phase errors estimated for the 4-frame Carre algorithm. Light intensity changes are assumed to have the contrast changes shown in Fig. 2. $I_{j}$ as sampled with phase shift $\alpha=0.5 \pi$.

Fig. 4: Phase errors estimated for the 4-frame Carre algorithm. Interference light intensity changes are assumed to have only contrast changes. These intensities are calculated by eq. (1) using $E=0.1$ and $N=0$. 
Fig. 5: Phase errors estimated for the 4-frame Carre algorithm. Light intensity changes are assumed to have contrast changes and noise. This intensities are calculated with eq. (1) using $E=0.1$ and $N=0.03$.

Fig. 6: Phase errors estimated for the simple 4-frame algorithm. Light intensity changes are calculated with eq. (1) using $E=0.1$ and $N=0.03$.

Fig. 7: Phase errors estimated for the 5-frame Hariharan algorithm of eq. (7b). Light intensity changes are calculated with eq. (1) using $E=0.1$ and $N=0.03$.

Fig. 8: Phase errors estimated for the 7-frame Groot algorithm of eq. (9b). Light intensity changes are calculated with eq. (1) using $E=0.1$ and $N=0.03$.

Fig. 9: Interference light intensities $I_{j}$ sampled around a contrast peak. The group of white circles is symmetric to the group of black circles. Phase of $I_{3}$ extracted by a required algorithm, has an opposite sign to the phase of $I_{3}^{\prime}$ extracted by the same algorithm. 
Fig. 10: Phase errors estimated by changing $c$ in eq. (16) with $b=d=0$ and phase shift $\alpha=0.7 \pi$. The solid curve represents $c=-1$ and the broken curve represents $c=0$, and the dotted curve represents $c=-2$.

Fig. 11: Phase errors estimated by changing $b$ and $d$ with $c=-1$ and phase shift $\alpha=0.7 \pi$. The solid curve represents $b=d=0$ : the 7 -frame algorithm, the broken curve represents $b=-5, d=0$, and the dotted curve represents $b=0, d=-4.5$. The broken and dotted curves have opposite-sign differences in the local area from the solid curve. The base lines not shown in the figure become less sloped compared with the solid curve.

Fig. 12: Phase errors estimated for eq. (16) using $c=-1$ and phase shift $\alpha=0.7 \pi$. The solid curve represents $b=d=0$ : the 7-frame algorithm, and the dotted curve represents $b=-22$, $d=-14.5$.

Fig. 13: Phase errors estimated with eq. (16) using $c=-1$ and phase shift $\alpha=0.3 \pi$. The solid curve represents $b=d=0$ : the 7-frame algorithm, and the dotted curve represents $b=23$, $d=14$. 
Fig. 14: Appropriate values for $b$ and $d$ which give the minimized phase errors for phase shift $\alpha$ between $0.3 \pi$ and $0.7 \pi$.

Fig. 15: Phase errors estimated with $b / d=1.6$ and $d$ given in Fig. 14. The inset figure is light intensity changes which are calculated by $E=0.1$ and $N=0.03$.

Fig. 16: Phase errors estimated with $b / d=1.6$ and $d$ given in Fig. 14. The inset figure is light intensity changes which are calculated by $E=0.15$ and $N=0.03$.

Fig. 17: Phase errors estimated for phase shift $\alpha$ between $6.3 \pi$ and $6.7 \pi$ using $b / d=1.6$ and $E=0.008$ and $N=0.03$. 


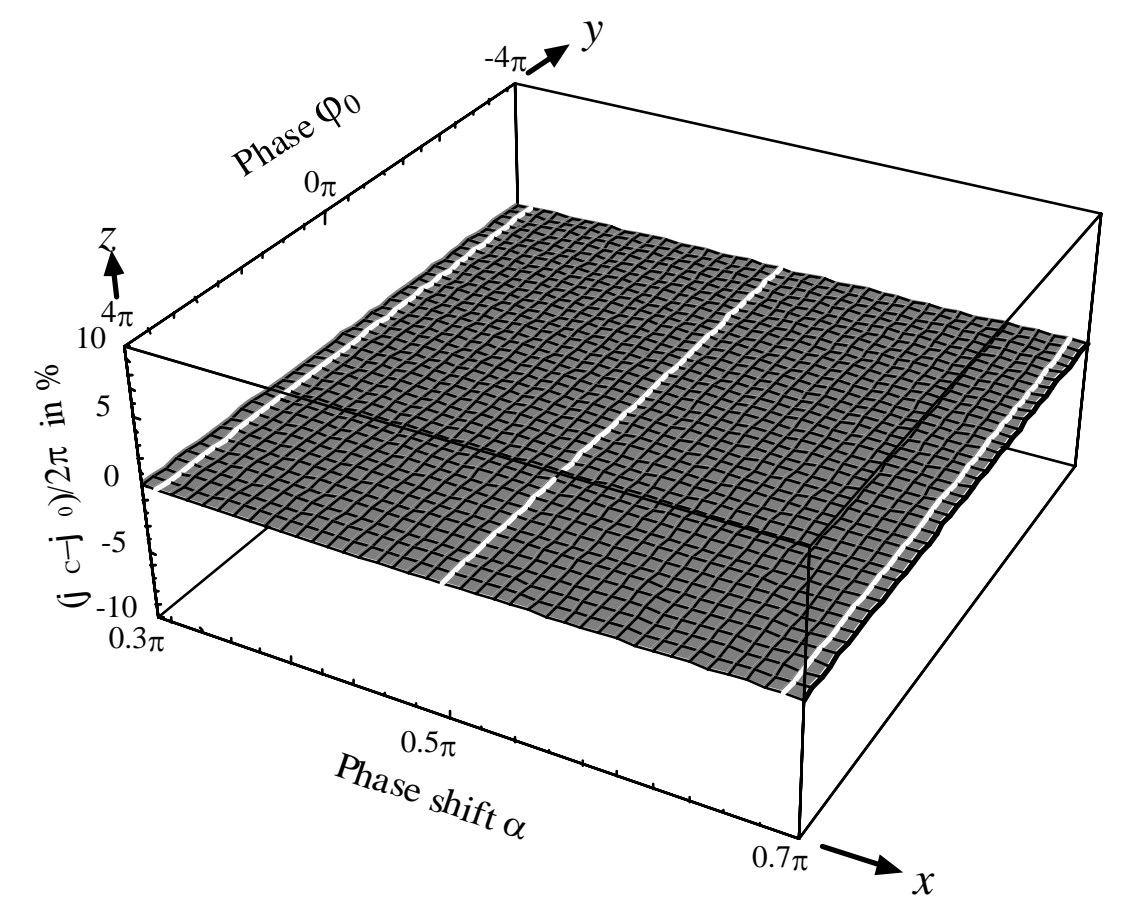

Fig.1 


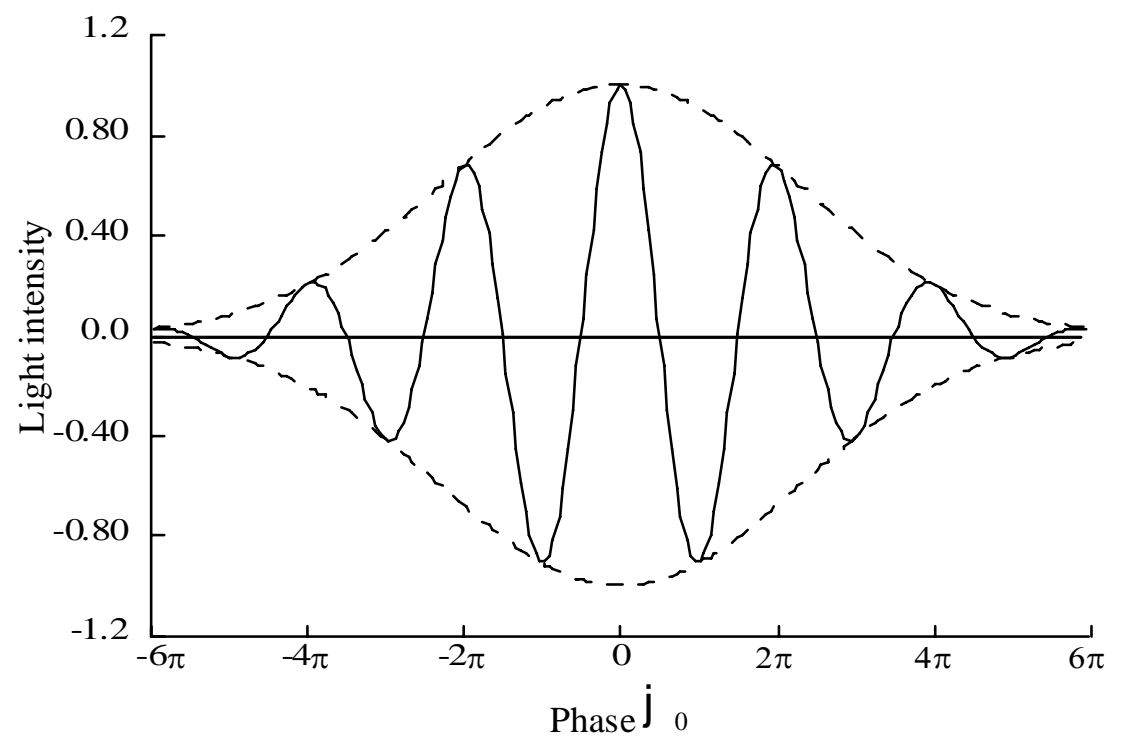

Fig.2 


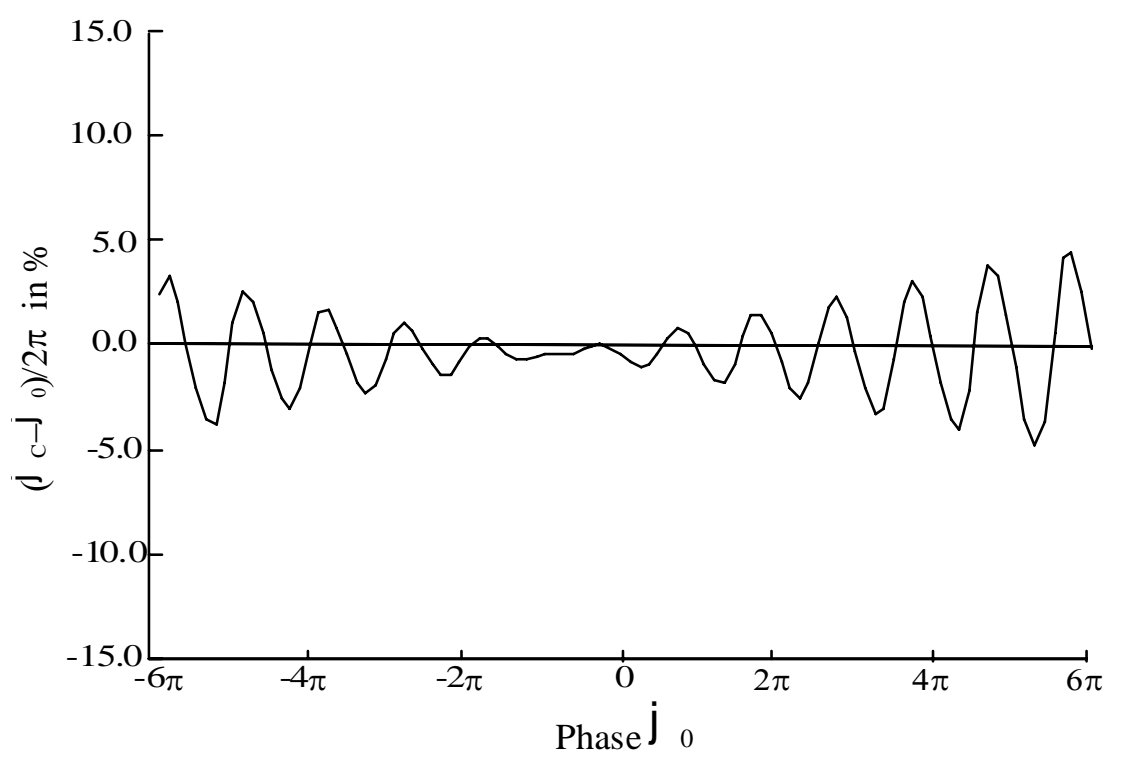

Fig.3 


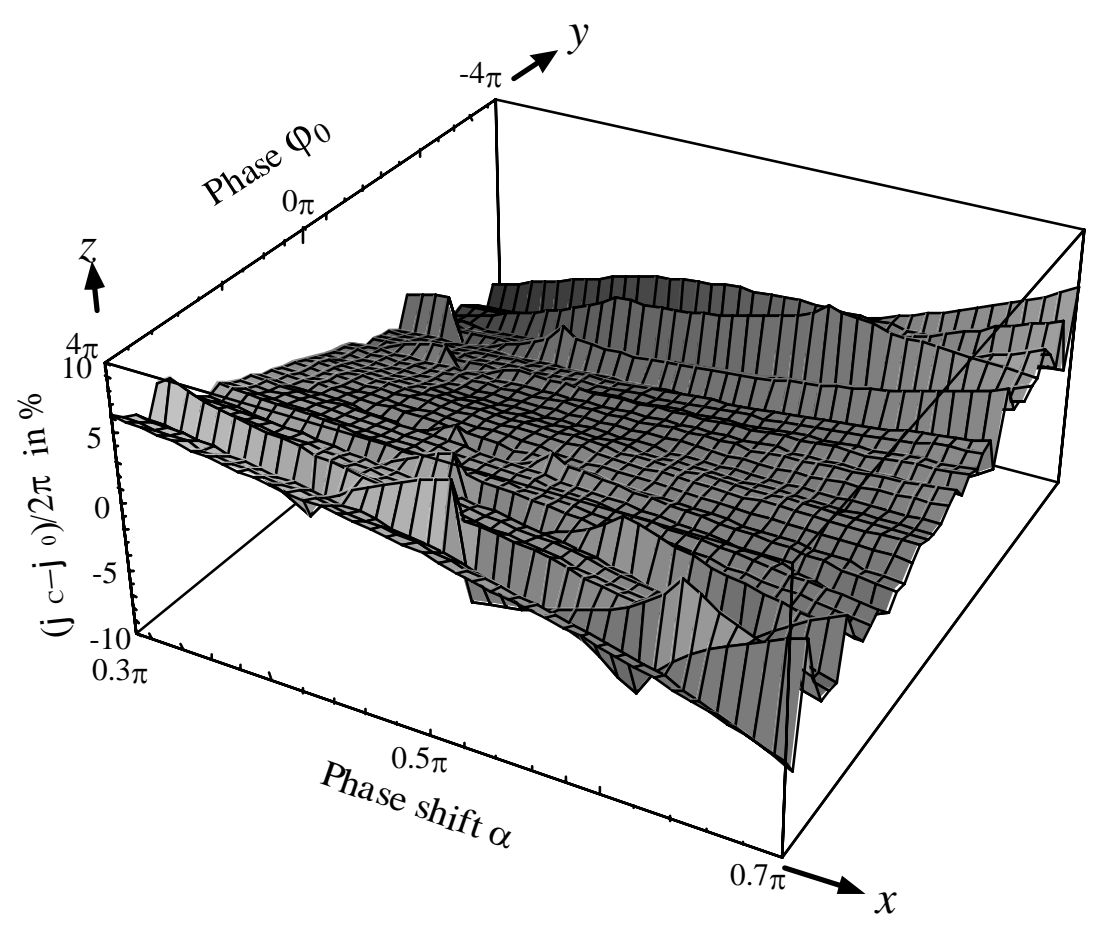

Fig.4 


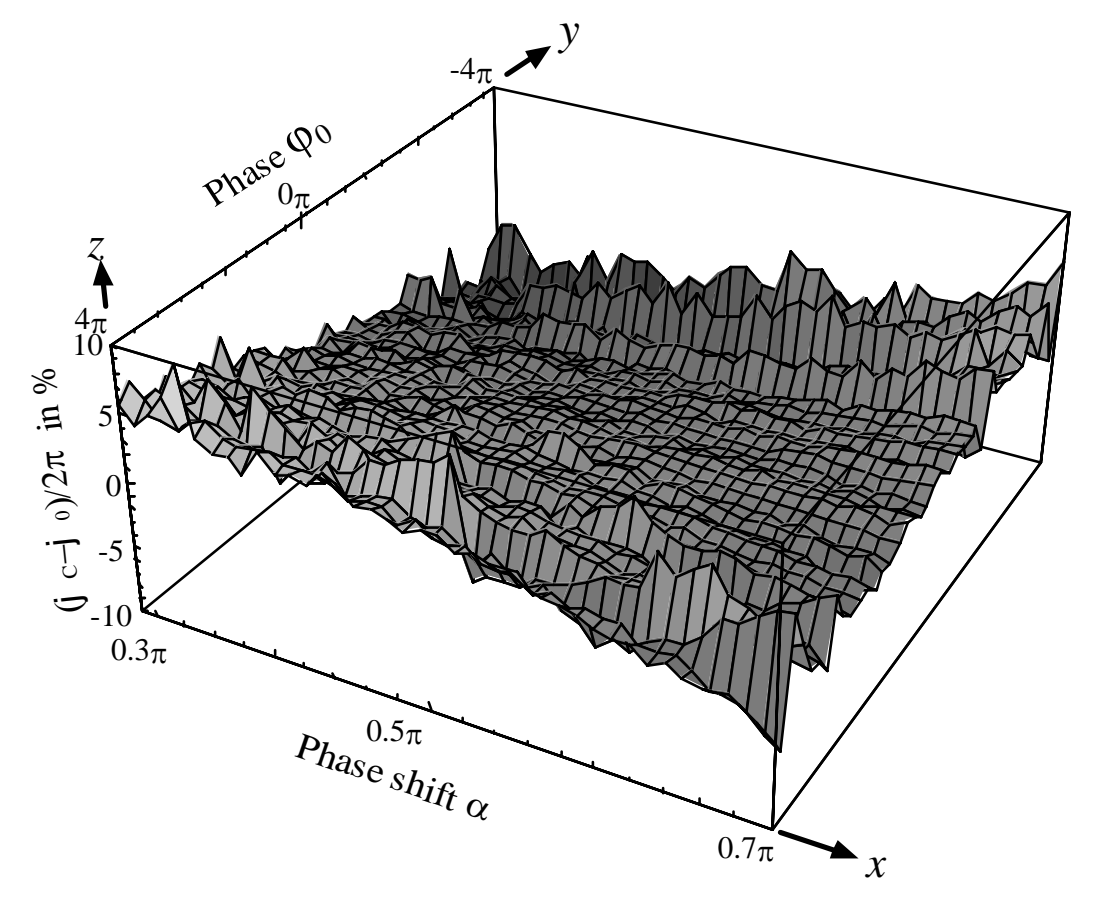

Fig.5 


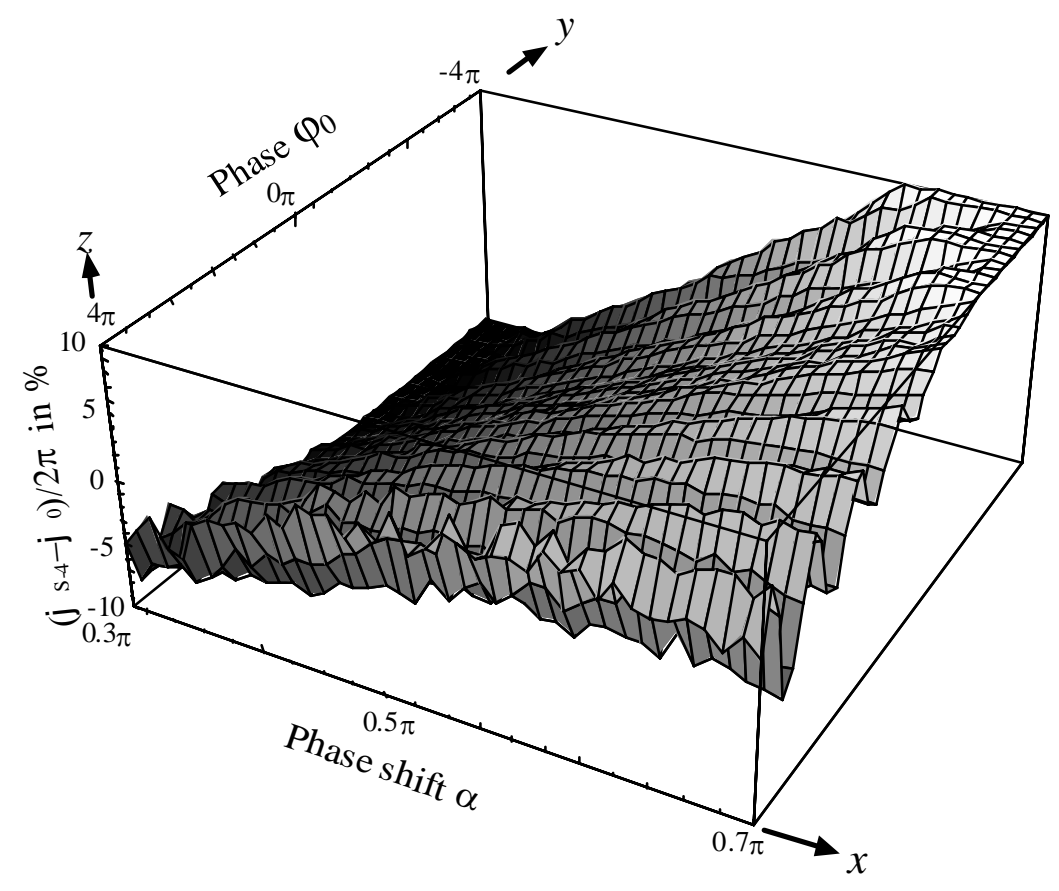

Fig.6 


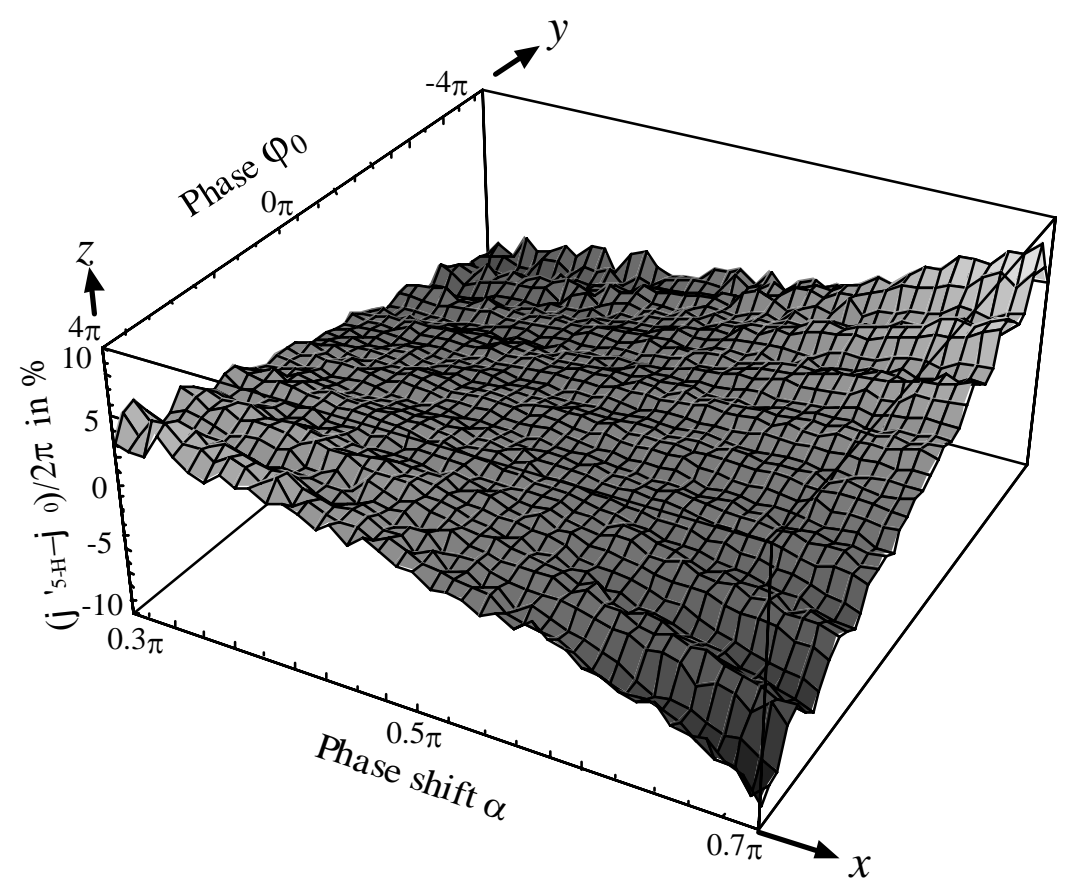

Fig.7 


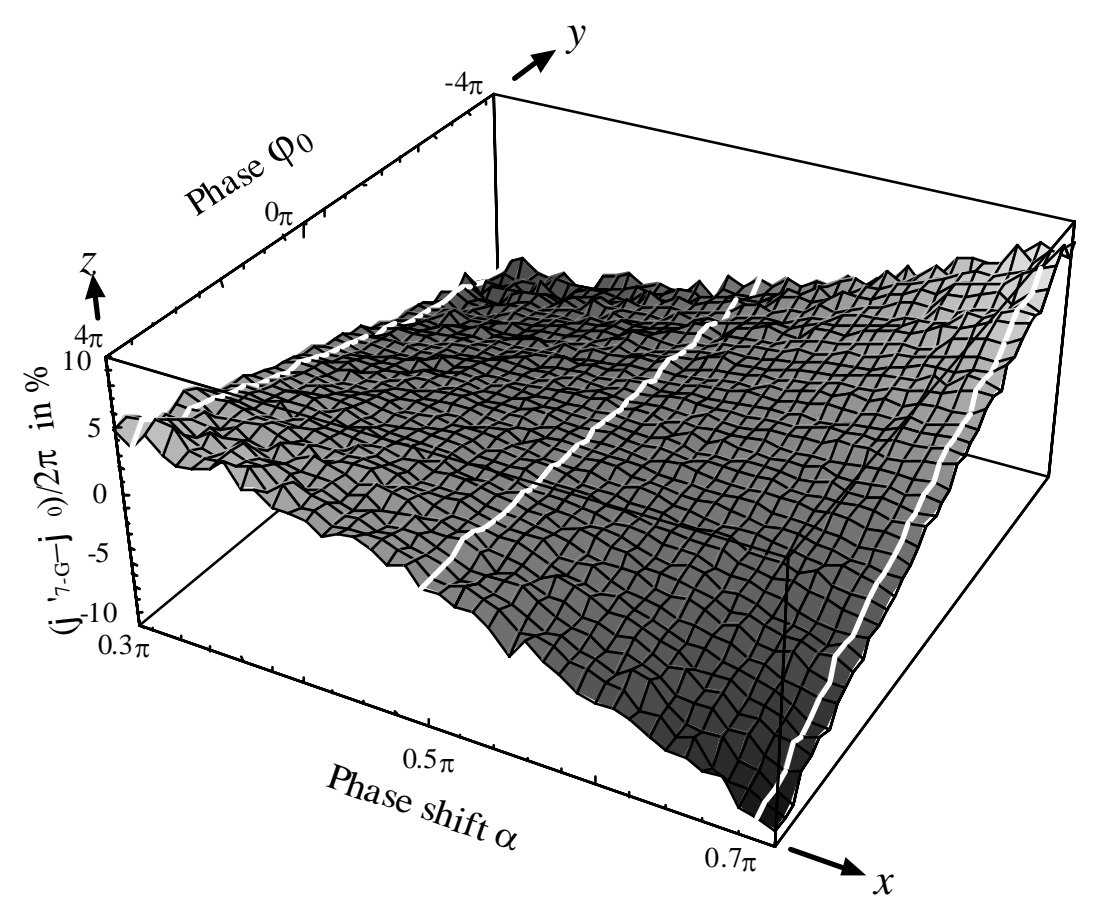

Fig.8 


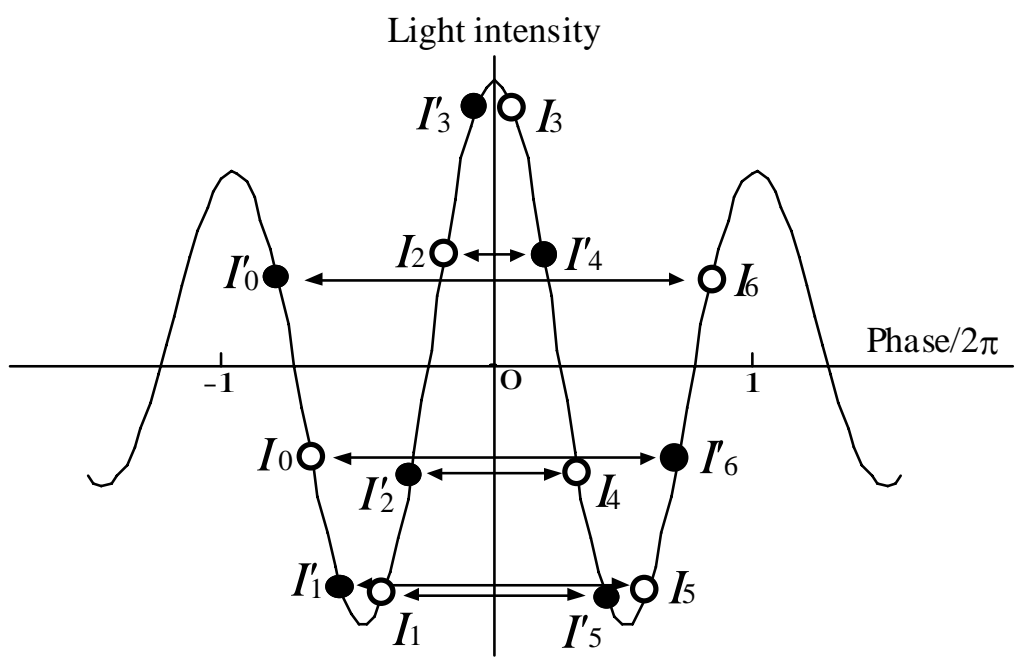

Fig.9 


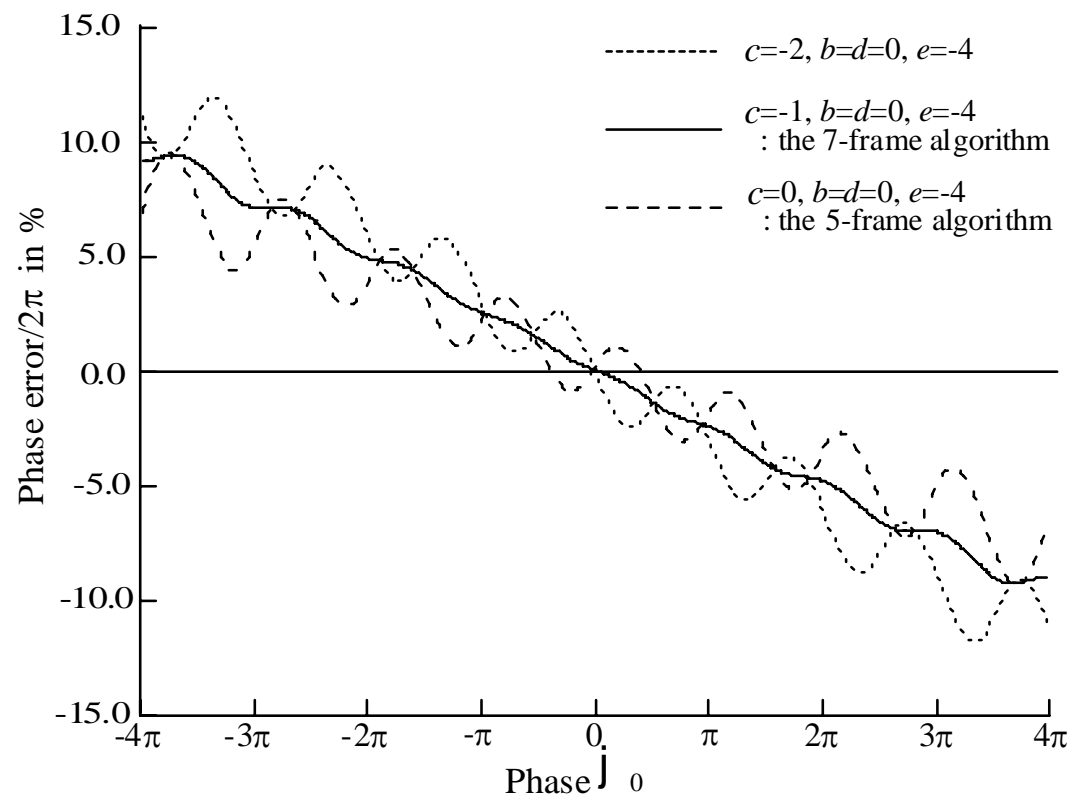

Fig.10 


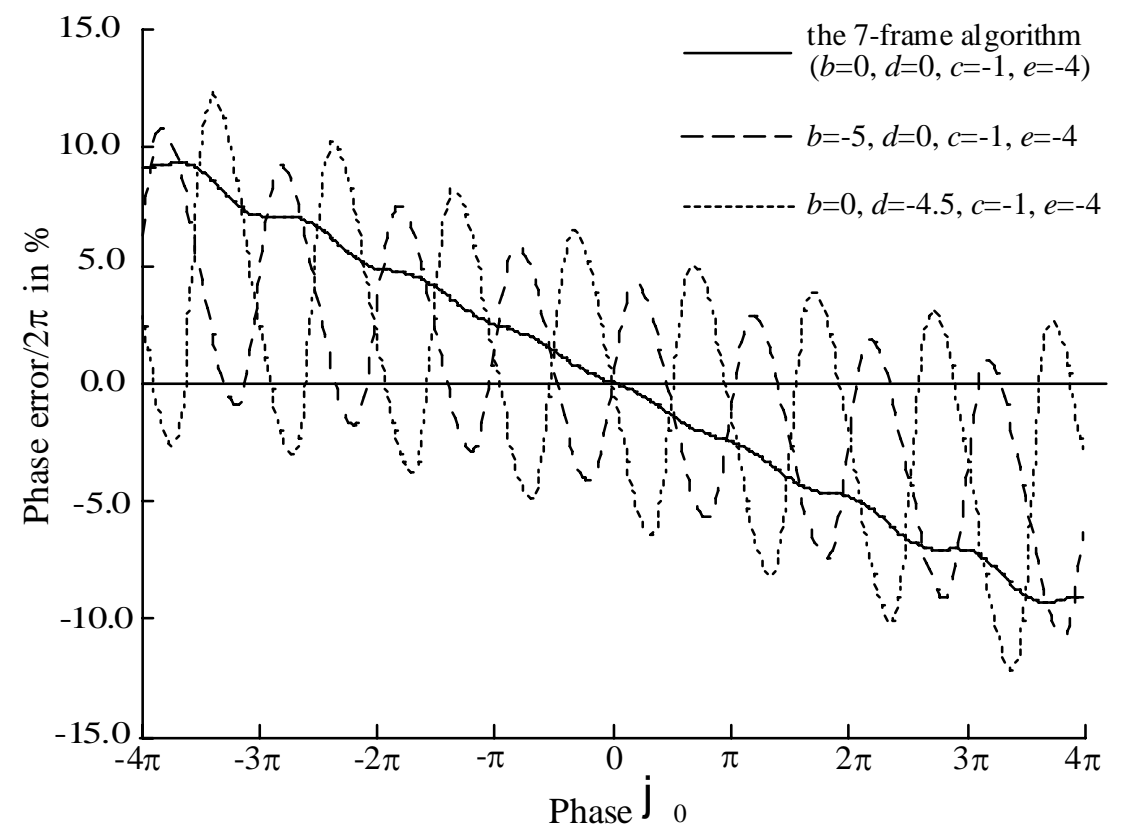

Fig.11 


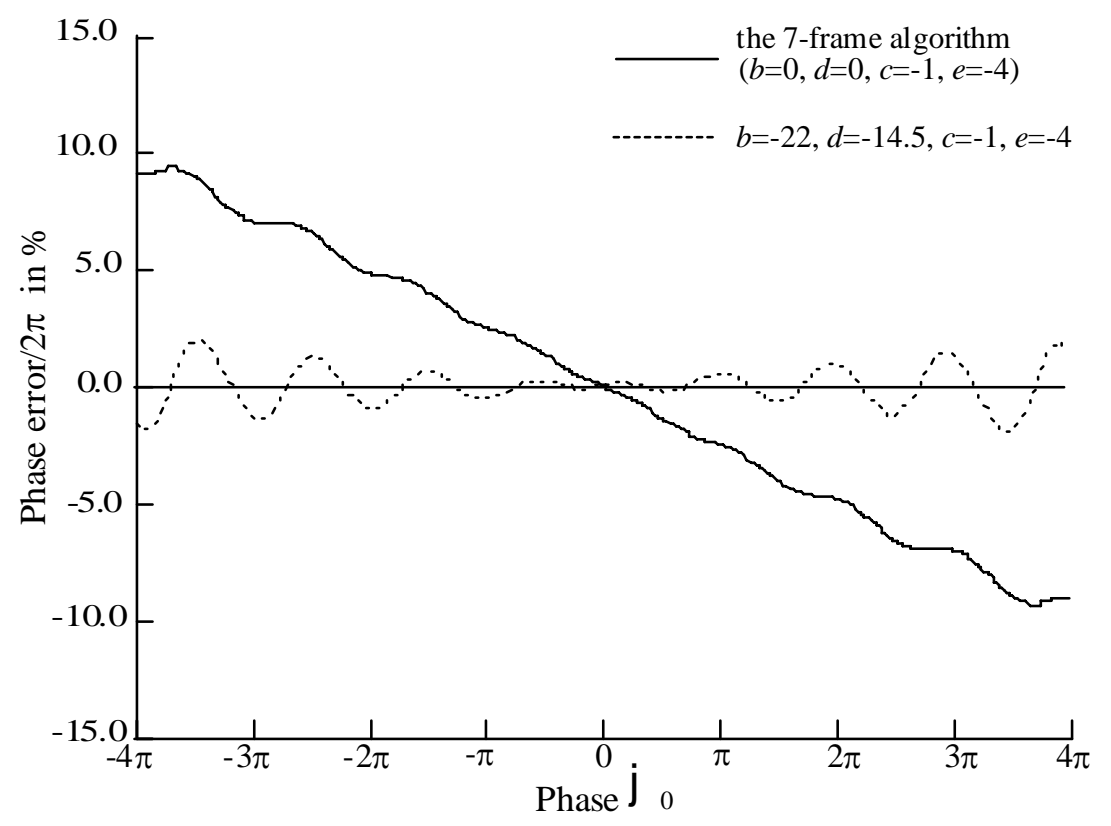

Fig.12 


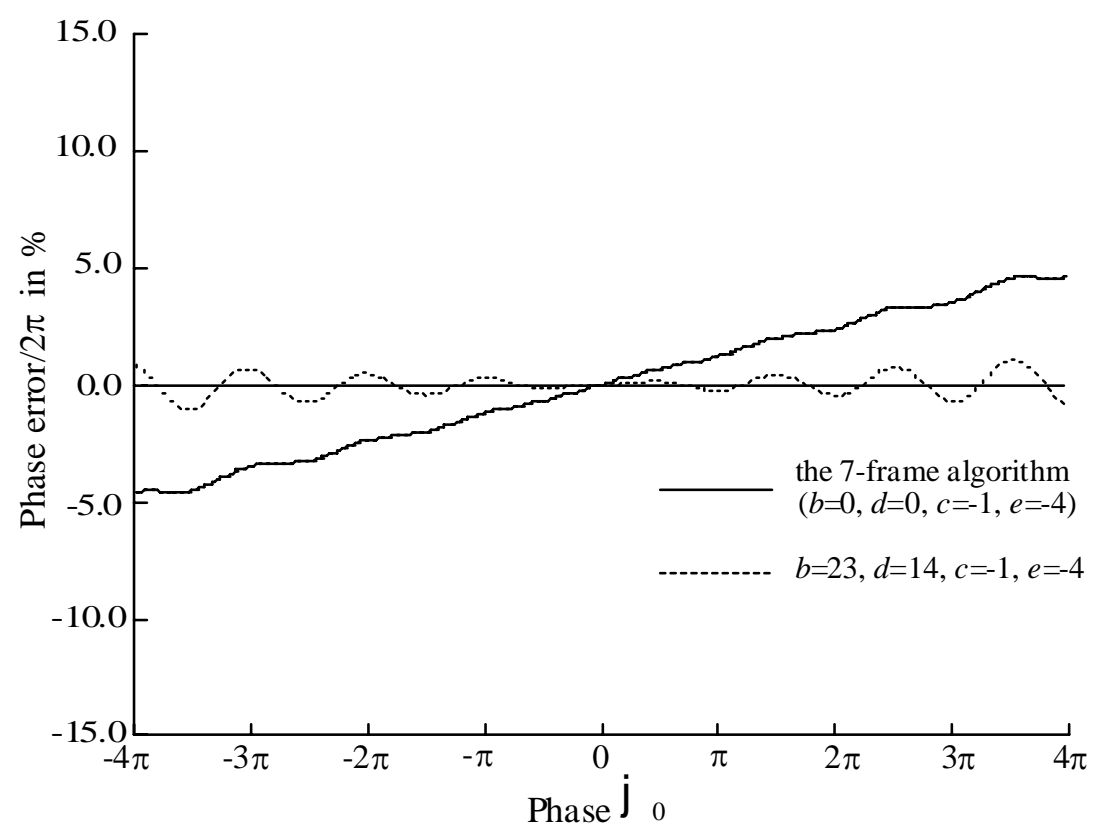

Fig.13 


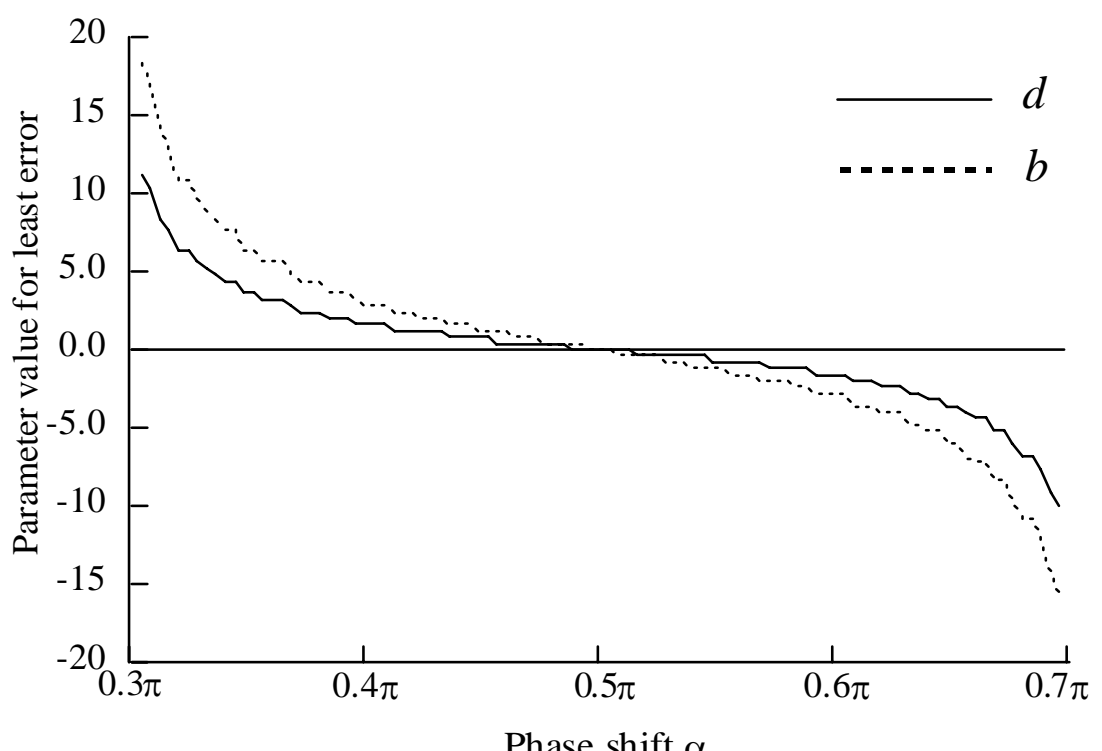

Phase shift $\alpha$

Fig.14 


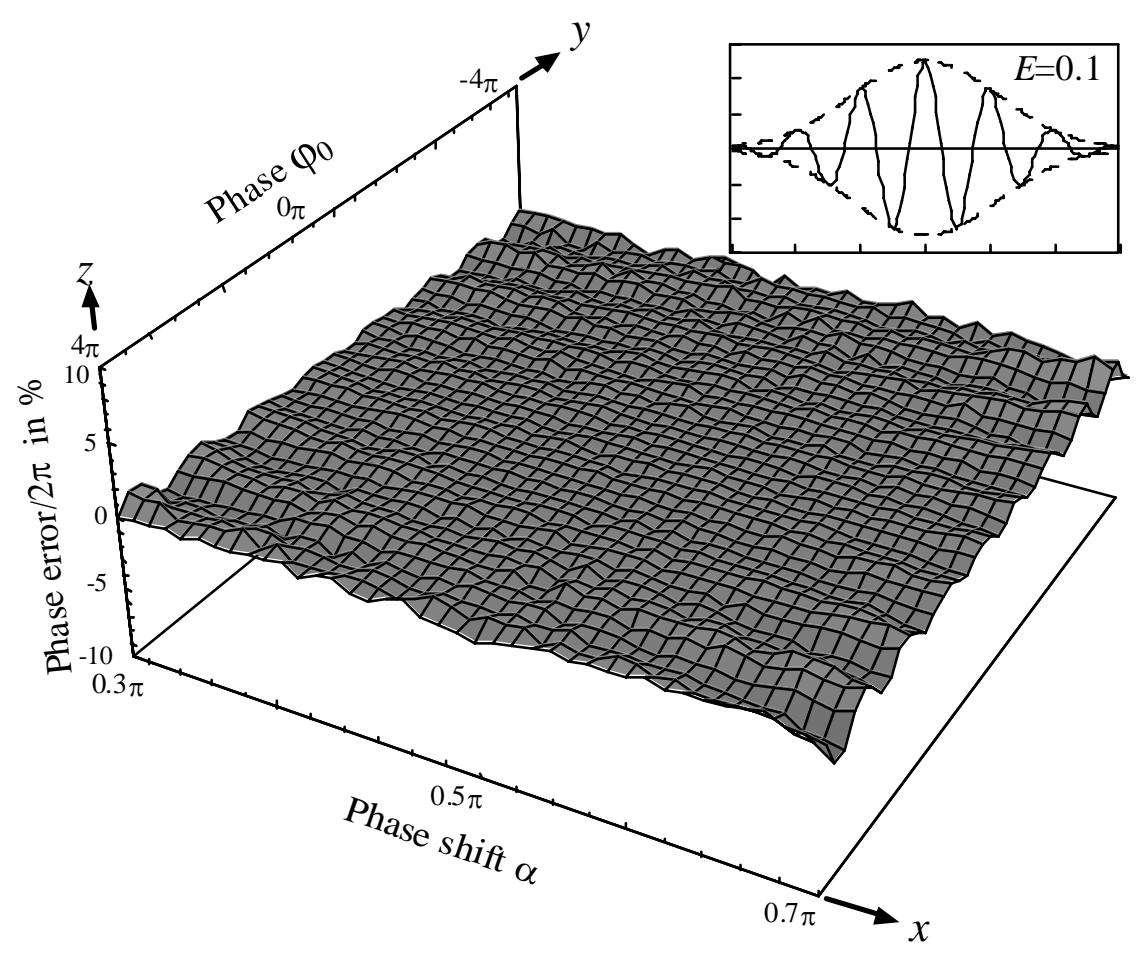

Fig.15 


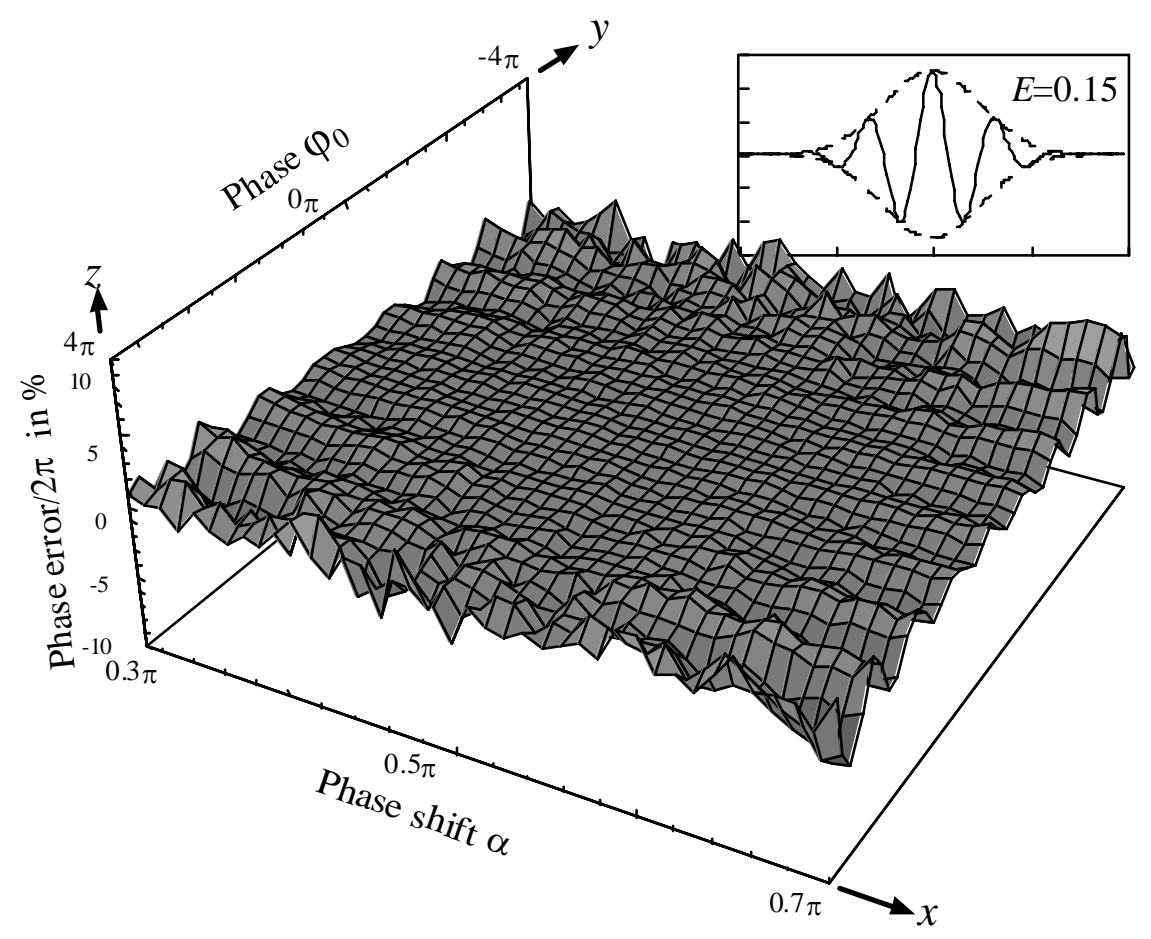

Fig.16 


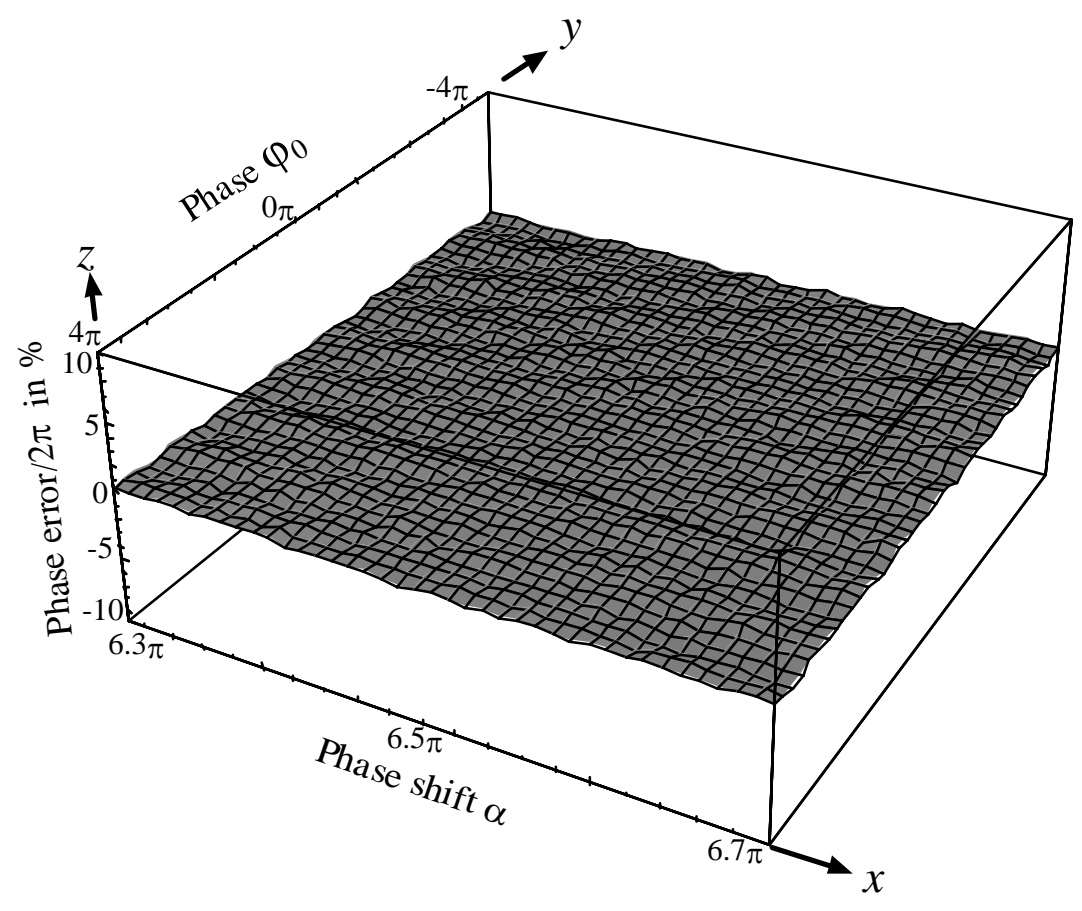

Fig.17 